\title{
Analysis and Evaluation of Regional Supply Chain Synergy
}

\author{
Liu Ziyu ${ }^{1}$, Xiao Jing $^{2}$ \\ ${ }^{1}$ Jilin Vocational and Technical Institute Communications, Changchun, 130012 \\ ${ }^{2}$ School of management, Changchun University, Changchun, 130022
}

Keywords: regional supply chain; synergy effect; factor analysis

Abstract: With the advent of the network economy era, regional supply chain synergy is an unavoidable problem and an indispensable part of regional economic development. This paper proposes the mechanism of regional supply chain collaborative development and obtains an effective approach to collaborative development based on questionnaire data, which provides a valuable reference for economic development in other regions.

\section{Introduction}

With the development of the information society, the competition between supply chains is becoming fiercer. The development of the network economy era has promoted the flat development of organizational management, coupled with the geographical distribution of enterprises, which requires supply chain work together between upstream and downstream to maximize benefits, optimize portfolios and rationalize collaboration configurations across the supply chain. This thesis intends to find the common factors of the development path of regional supply chain by designing the principal-supply chain coordination model and solving and analyzing the model. According to the synergy effect, the paper finds the effective path of regional supply chain development and provides valuable guidance for the development of regional supply chain.

\section{Factor Analysis on Mechanism of Regional Supply Chain Development Synergy}

According to Xu Weiyang (2015) of Beijing Jiaotong University to analyze the theory of supply chain, the model can be divided into subsidiary enterprise utility and market sales volume model. The final deterministic equivalent income of affiliated enterprises is:

$$
I=\alpha+\beta(p-c)(A x+B)-\frac{b x^{2}}{2}-\frac{1}{2} r(p-c)^{2} \beta^{2} \sigma^{2}
$$

And $r(p-c)^{2} \beta^{2} \sigma^{2}$ is risk cost. The leading company's expected return model is:

$$
\mathrm{E}(\mathrm{U})=\mathrm{E}((\mathrm{p}-\mathrm{c}) \mathrm{Q}-\mathrm{s}(\mathrm{Q}))=\mathrm{E}((\mathrm{p}-\mathrm{c}) \mathrm{Q}-\alpha-\beta(\mathrm{p}-\mathrm{c}) \mathrm{Q})=-\alpha+(\mathrm{p}-\mathrm{c})(1-\beta)(\mathrm{Ax}+\mathrm{B})
$$

The agency costs of leading companies are:

$$
\mathrm{TC}=\mathrm{R}+\left(\mathrm{E}\left(\mathrm{O}_{1}\right)-\mathrm{E}\left(\mathrm{C}_{1}\right)\right)
$$

The incentive model that can establish the coordinated development of regional supply chain 
through analysis is:

$$
\alpha+\beta(p-c)(A x+B)-\frac{b x^{2}}{2}-\frac{1}{2} r(p-c)^{2} \beta^{2} \sigma^{2} \geq \underline{s}
$$

Therefore, the regional supply chain incentive synergy model consisting of leading companies and affiliated companies is:

$$
\begin{gathered}
\mathrm{E}(\mathrm{U})=\max \{-\alpha+(1-\beta)(\mathrm{p}-\mathrm{c})(\mathrm{Ax}+\mathrm{B})\} \\
\text { s.t. }\left\{\begin{array}{c}
\operatorname{argmax}\left\{\alpha+\beta(\mathrm{p}-\mathrm{c})(\mathrm{Ax}+\mathrm{B})-\frac{1}{2} \mathrm{r}(\mathrm{p}-\mathrm{c})^{2} \beta^{2} \sigma^{2}-\frac{\mathrm{bx}^{2}}{2}\right. \\
\alpha+\beta(\mathrm{p}-\mathrm{c})(\mathrm{Ax}+\mathrm{B})-\frac{\mathrm{bx}^{2}}{2}-\frac{1}{2} \mathrm{r}(\mathrm{p}-\mathrm{c})^{2} \beta^{2} \sigma^{2} \geq \underline{s}
\end{array}\right.
\end{gathered}
$$

Under the condition of information symmetry, $x_{1}^{*} \approx x^{*}=\frac{A \beta(p-c)}{b}, \beta_{1}^{*} \approx \beta^{*}=0$, the model conclusion is amended to:

$$
\alpha_{1}^{*} \approx \alpha^{*}=\mathrm{s}+\frac{\mathrm{A}^{2}(\mathrm{p}-\mathrm{c})^{2}}{2 \mathrm{~b}}
$$

Under information asymmetry, $x=\frac{A \beta(p-c)}{b}, \beta_{1}=\frac{A^{2}-B b}{2 A^{2}}, \beta_{2}=\frac{B b}{r b \sigma^{2}-A^{2}}$. The agency costs of leading companies are:

$$
\mathrm{TC}=\mathrm{R}+\mathrm{E}(\mathrm{L})=(\mathrm{p}-\mathrm{c})^{2} \mathrm{r} \sigma^{2} \frac{1+\mathrm{rb} \sigma^{2} / \mathrm{A}^{2}}{2\left(1+\frac{\mathrm{rb} \sigma^{2}}{\mathrm{~A}^{2}}\right)^{2}}=(\mathrm{p}-\mathrm{c})^{2} \mathrm{r} \sigma^{2} \frac{1}{2\left(1+\mathrm{rb} \sigma^{2} / \mathrm{A}^{2}\right)}
$$

\section{Empirical Analysis of Regional Supply Chain Synergies}

\subsection{Indicator design and research hypothesis proposed}

This paper uses a second questionnaire survey method. The first questionnaire is used to investigate the importance of factors. There are 30 options and each respondent should choose 17 of them as factors of their own concern. A total of 330 questionnaires were distributed. 325 copies

\begin{tabular}{|c|c|c|c|c|c|}
\hline point of view & Index & Number & Frequency & ratio & Attention \\
\hline \multirow{4}{*}{ Subsidiary capacity level } & Degree of technical application & 300 & 76 & $3.82 \%$ & $37.3 \%$ \\
\hline & Service Level & 300 & 176 & $8.85 \%$ & $86.3 \%$ \\
\hline & Capital abundance & 300 & 97 & $4.88 \%$ & $47.6 \%$ \\
\hline & company culture & 300 & 90 & $4.39 \%$ & $42.72 \%$ \\
\hline \multirow{4}{*}{ Effort cost factor } & Managerial competence level & 300 & 34 & $6.74 \%$ & $66 \%$ \\
\hline & Employee competence level & 300 & 56 & $2.82 \%$ & $27.5 \%$ \\
\hline & Advanced level of existing facilities & 300 & 78 & $3.92 \%$ & $38.2 \%$ \\
\hline & Master the advanced level of technology & 300 & 156 & $7.85 \%$ & $76.5 \%$ \\
\hline \multirow{3}{*}{ Absolute risk aversion } & Policy assurance and support & 300 & 145 & $7.29 \%$ & $7.1 \%$ \\
\hline & Business size & 300 & 89 & $4.48 \%$ & $43.6 \%$ \\
\hline & Incentive level & 300 & 133 & $6.69 \%$ & $65.2 \%$ \\
\hline \multirow{3}{*}{ Minimum income level } & Rationality of distribution & 300 & 204 & $10.26 \%$ & $100 \%$ \\
\hline & Corporate market position & 300 & 153 & $7.7 \%$ & $75 \%$ \\
\hline & opportunity cost & 300 & 64 & $3.22 \%$ & $31.4 \%$ \\
\hline \multirow{3}{*}{ Output stability } & Risk control experience and technology & 300 & 155 & $7.8 \%$ & $76 \%$ \\
\hline & Emergency management capability & 300 & 98 & $4.93 \%$ & $48 \%$ \\
\hline & Institutional perfection & 300 & 87 & $4.38 \%$ & $42.7 \%$ \\
\hline
\end{tabular}
were recovered and 300 valid questionnaires were valid. The effective rate was $90.9 \%$. In this paper, the top 17 indicators of attention are used as the final secondary survey data items (the indicators after ranking 18 are not shown in the table below).

Table 1 Variable influencing factors 
Based on the research basis and the actual survey situation, the project proposes the following research hypothesis (Figure 1).

$\mathrm{H} 1$ the improvement of the level of competence of affiliated enterprises contributes to the reduction of the cost factor of their efforts; $\mathrm{H} 2$ an increase in the level of competence of affiliates can reduce the variance of their output; H3 the improvement of the ability level of affiliates can reduce their absolute risk aversion; $\mathrm{H} 4$ the increase in the cost factor of affiliates will increase their minimum income level; $\mathrm{H} 5$ reduces the effort cost factor of affiliates to help reduce their absolute risk aversion; $\mathrm{H} 6$ raising the minimum income level of affiliates will increase the level of variance in their output; $\mathrm{H} 7$ reduces the minimum income level of affiliates to increase their absolute risk aversion; $\mathrm{H} 8$ an increase in the absolute risk aversion of will increase the variance of its output.

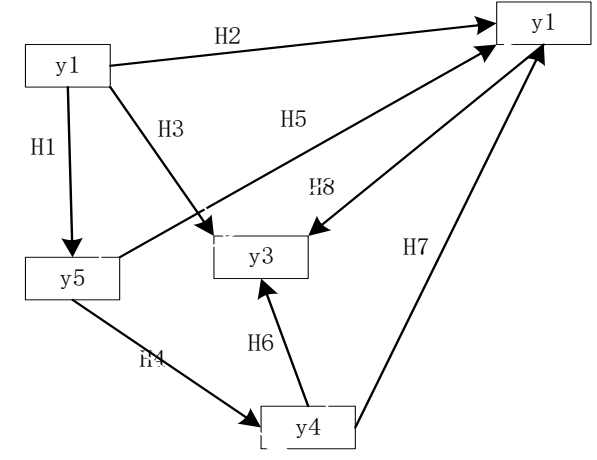

Figure 1 study hypothesis

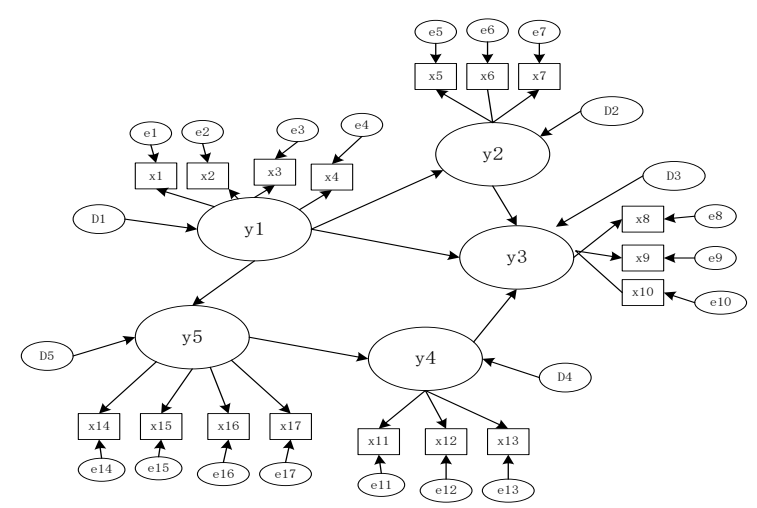

Figure 2 variables and variable structure

$\mathrm{y}_{1}$ : ability level of affiliated companies, $\mathrm{y}_{2}$ : absolute risk aversion, $\mathrm{y}_{3}$ : output variance, $\mathrm{y}_{4}$ : minimum return level, $\mathrm{y}_{5}$ : effort cost factor.

\subsection{Summary of the questionnaire}

The second survey began on March 10, 2018, and the deadline was May 6, 2018. The survey targets 1300 managers of 112 companies in the transportation, warehousing, postal industries, wholesale, retail and trade supply chains in Jilin Province. This questionnaire is used to investigate the development path of the supply chain and has 17 questions. According to simple random sampling, 1300 copies of this questionnaire were distributed, 1,240 copies were recovered, and 1,180 valid questionnaires were valid, whose effective rate is $90.8 \%$.

According to Figure 2, the exogenous variables $\mathrm{x}_{1}, \mathrm{x}_{2}, \mathrm{x}_{3}$, and $\mathrm{x}_{4}$ represent the degree of technology application, service level, capital adequacy, and corporate culture. The endogenous variables $\mathrm{X}_{5}-\mathrm{X}_{17}$ represent policy assurance and support, the size of the enterprise, Incentive mechanism level, allocation rationality, enterprise market position, opportunity cost, risk control experience and technology, emergency management capability, system perfection level, management personnel ability level, staff ability level, advanced level of existing facilities and advanced technology Level. Exogenous latent variable $\mathrm{y}_{1}$ indicates the ability level of affiliated enterprises, and endogenous latent variables $\mathrm{y}_{2}, \mathrm{y}_{3}, \mathrm{y}_{4}$ and $\mathrm{y}_{5}$ belong to absolute risk aversion, output variance, minimum return level and effort cost coefficient. There are residuals in the explicit variables, and there are variances in the exogenous latent variables and endogenous latent variables.

After the model running, the fits of chi-square/degree of freedom, RMSEA, NFI, PNFI, GFI, AGFK PGFI, TLI, and CFI are shown in Table 2. 
Table 2 Fitting (1)

\begin{tabular}{|c|c|c|c|c|c|c|c|c|c|}
\hline Index & X2/df & RMSEA & NFI & PNFI & PGFI & GFI & AGFI & TLI & CFI \\
\hline Model & 1.866 & 0.054 & 0.928 & 0.512 & 0.466 & 0.950 & 0.898 & 0.934 & 0.964 \\
\hline Reception & $1-5$ & $<0.08$ & $>0.9$ & $>0.5$ & $>0.5$ & $>0.9$ & $>0.9$ & $>0.9$ & $>0.9$ \\
\hline
\end{tabular}

Since Chi-square is too large and has a significant level of significance, it is necessary to refer to the result of the correction index $(\mathrm{Ml}>5)$ to correct the model, which increases the degree of correlation between variables and reduces the degree of freedom and Chi-square value. After the model re-running, the following results are obtained (Table 3):

Table 3 Fitting (2)

\begin{tabular}{|c|c|c|c|c|c|c|c|c|c|c|}
\hline Index & X2/df & P & RMSEA & NFI & PNFI & PGFI & GFI & AGFI & TLI & CFI \\
\hline Model & 1.439 & 0.00 & 0.038 & 0.948 & 0.488 & 0.441 & 0.963 & 0.920 & 0.967 & 0.983 \\
\hline Reception & $1-5$ & - & $<0.08$ & $>0.9$ & $>0.5$ & $>0.5$ & $>0.9$ & $>0.9$ & $>0.9$ & $>0.9$ \\
\hline
\end{tabular}

It can be seen from Table 3 that the $\mathrm{X}_{2}$ value becomes smaller, $\mathrm{P}$ value becomes larger, and the $\mathrm{X} 2 / \mathrm{df}$ is 1.439 , which is within the acceptance range. Therefore, the overall fitness of the model can be considered to pass the test. Other statistical indicators are statistically significant. For example, the RMSEA value is 0.038, less than 0.08. The NFI/GFI/AGFI/TLI and CFI values all exceed the critical value of 0.9 , and the PNFI and PGFI values are also approximately greater than 0.5 . The model is therefore tested by relative fit.

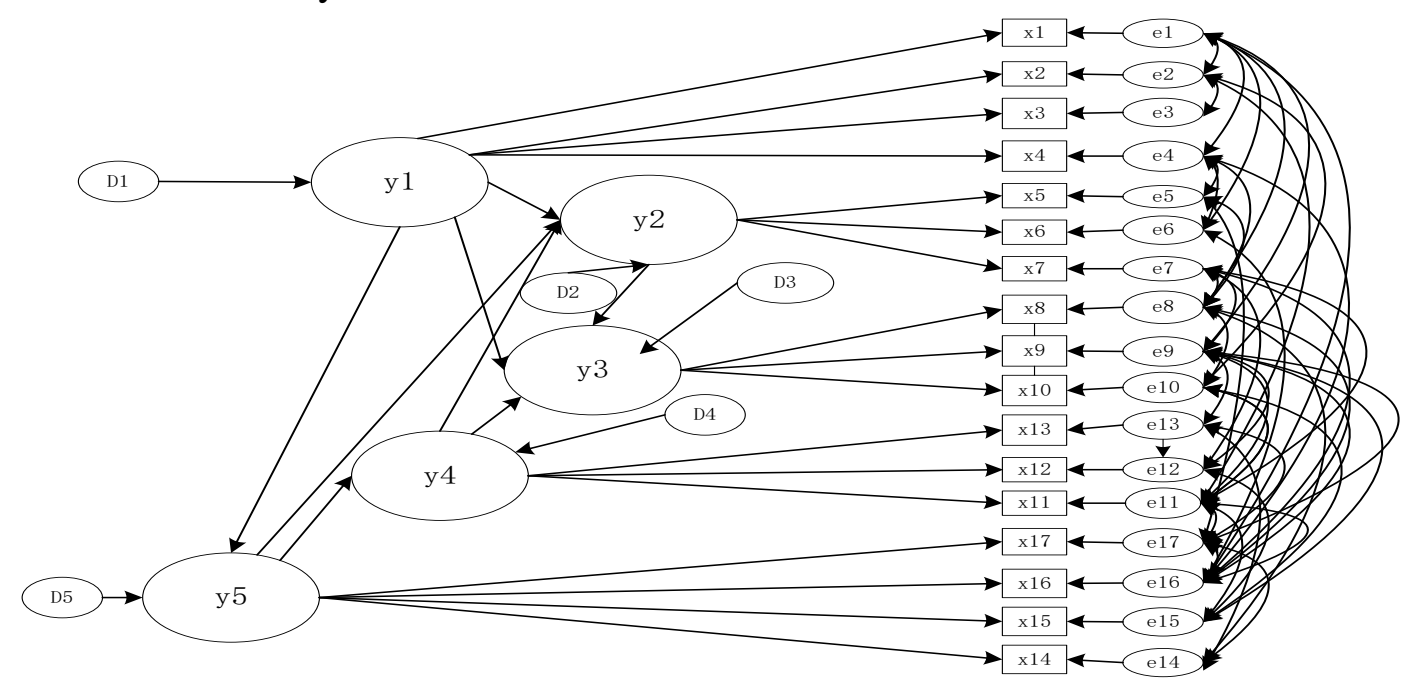

Figure 3 modified model

\section{Conclusions}

In the results of running the modified model, Estimate represents the estimated path coefficient, and $\mathrm{CR}$ represents the $\mathrm{t}$ value. According to the hypothesis test condition, the $\mathrm{t}$ value needs to be greater than 1.96 to support the null hypothesis (significant relationship). $\mathrm{P}$ represents the significance level, and The $\mathrm{P}$ value needs to be less than 0.05 to support the null hypothesis.

Through analysis, affiliates can use their information superiority and conceal relevant self-interested information to obtain benefits. Subsidiary enterprise capability level, effort cost coefficient, absolute risk aversion degree, output variance and minimum income level variable are important factors affecting the coordinated development of regional supply chain. The ability level of the affiliated enterprises is most directly reflected in its system perfection level, technical level, 
service level, scale, capital adequacy and corporate culture. Therefore, the leading enterprises should provide support and system easing in all aspects of the affiliated enterprises. The cost of efforts of affiliated companies can be understood to the same extent as the level of competence. The cost of efforts is directly reflected in the size of affiliated companies, the level of competence of managers and employees, the advanced level of existing facilities, and the advanced level of technology. The cost coefficient of effort reflects the cost of the product of a certain level of effort provided by the subsidiary company. The cost unit of the affiliated company is directly related to the final benefit. The effort cost is low, indicating that the company's ability to provide products is greater, and could gain an advantage in market competition. Affiliated enterprises could use their own low cost coefficient to cope with market competition, and leading enterprises can guide the training of affiliated industry personnel, qualification examinations and the improvement of facilities and technology level, which could improve the quality of affiliated enterprises and employees and ensure healthy development of regional supply chains.

\section{References}

[1] Gong Daqing, Liu Shifeng, Wang Yueping. Research on Entrustment Agency of Supply Chain Incentive Mechanism under Logistics Resource Integration Environment [J].Soft Science, 2013, 27(5): 51-55, 65.

[2] Xu W.Y., Zhang Z.J., Gong D.Q. Cooperative Supply Chain Management under Asymmetric Information [J] Joumal of Applied Research and Technology, 2014.

[3] Du Bin. Research on the interaction between regional logistics and wholesale and retail trade in Linyi City [D]. Beijing: Beijing Jiaotong University, 2013.

[4] Xu Weiyang, Zhang Zhenji, Gong Daqing, Du Bin. Case Study on Regional Logistics and Trade Relationship between Zero and Zero [J]. Economic Aspects, 2014(8): 69-72. 